\title{
Robotic arm joint position control using iterative learning and mixed sensitivity $\mathrm{H} \infty$ robust controller
}

\author{
Petrus Sutyasadi, Martinus Bagus Wicaksono \\ Mechatronics Department, Politeknik Mekatronika Sanata Dharma, Indonesia
}

\begin{tabular}{l}
\hline \hline Article Info \\
\hline Article history: \\
Received Dec 30, 2020 \\
Revised Mar 6, 2021 \\
Accepted Jun 20, 2021 \\
\hline
\end{tabular}

Keywords:

$\mathrm{H} \infty$ robust controller Iterative learning controller Robotic arm SCARA robot

\begin{abstract}
This paper proposes an improved control strategy of a robotic arm joint using hybrid controller consist of $\mathrm{H} \infty$ robust controller and iterative learning controller. The main advantage of this controller is the simple structure that made it possible to be implemented on a small embedded system for frugal innovation in industrial robotic arm development. Although it has a simple structure, it is a robust $\mathrm{H} \infty$ controller that has robust stability and robust performance. The iterative learning controller makes the trajectory tracking even better. To test the effectiveness of the proposed method, computer simulations using Matlab and hardware experiments were conducted. Variation of load was applied to both of the processes to present the uncertainties. The superiority of the proposed controller over the proportional integral derivative (PID) controller that usually being used in a low-cost robotic arm development is confirmed that it has better trajectory tracking. The error tracking along the slope of sinusoidal trajectory input was suppressed to zero. The biggest error along the trajectory that happened on every peak of the sinusoidal input, or when the direction is changed has been improved from 15 degrees to 4 degrees. This can be conceived that the proposed controller can be applied to control a low-cost robotic arm joint position which is applicable for small industries or educational purpose.
\end{abstract}

This is an open access article under the CC BY-SA license.

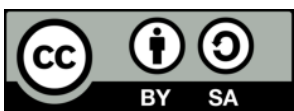

\section{Corresponding Author:}

Petrus Sutyasadi

Mechatronics Department

Politeknik Mekatronika Sanata Dharma

Paingan, Maguwoharjo, Depok, Sleman 55283, Indonesia

Email: peter@pmsd.ac.id

\section{INTRODUCTION}

In this era of industry 4.0, maximum efficiency with no downtime production line become the dream of every company [1]. Robot is one good candidate to make it happen. In manufacturing, human labor was said to be less practical than industrial robot [2]. As long as the robots are maintained properly, they can do their job tirelessly, precisely, and consistently. That is why many industries adopt industrial robots in their line of production. The robot market has grown significantly around 15 percent from 2014 to 2017 and predicted it will be more for the next decades [3]. Despite its great contribution to productivity, the cost of a robot system is very expensive. Small to medium industries will not easily employ robots in their production systems. Their problem will be solved if the cost of an industrial robot is not so expensive. If small to medium industries have opportunities to use robots in their production, they will need human resources to operate and maintain the robot. Therefore, low-cost industrial robots for universities or vocational school is also important. Because the schools produce skilled human resources that support the use of the robots in 
industries. Universities or vocational schools need more low-cost industrial robotic arms in their educational process. It saves more budget if the robot can be provided locally.

There are many published studies about designing and developing low-cost robotic arms. Besides the price that is lower than the commercial one, low-cost robot components are easily found in the market [4]. Due to the constraints from the component availability in the market, a low-cost robot can have a different architecture from time to time [4] and have less performance than the commercial one [5]. The overall performance can be optimized using a better control algorithm. However, since the embedded system being used is also a small and a low-cost controller, the control algorithm should be a low order controller that has a simple structure. A considerable amount of literature has been published on the issue of a simple controller for a small robotic arm.

Some researchers have developed low-cost robotic arm controller using a simple controller such as proportional integral (PI) controller [6], PID [7]-[9], fuzzy logic [10], [11], hybrid PID, and fuzzy [12], [13], optimal controller [14], neural network [15] and model-based controller [16]. A few researchers also tried to use robust control to control robotic arm joints such as sliding mode controller [17]-[20] and hybrid PIDsliding mode controller [21]. However, all of those researches were done on simulation or implemented on a very small robotic arm using small radio control (RC) servo motors. Nothing was done on a real size robotic arm that considerably applicable in small to medium industries. Secondly, mostly their experiment were done with a step command and were not investigated with a specific trajectory for example sinusoidal trajectory. Thirdly, none of them showed robustness among load variation which is commonly happened on a robotic arm that handles many different tasks.

This paper proposes a hybrid controller to control robotic arm joints using reduced order mixedsensitivity $\mathrm{H} \infty$ combined with iterative learning controller. To guarantee good trajectory tracking, iterative learning controller (ILC) was implemented. ILC needs a condition that the system should be a stable closedloop system before implementing the ILC. Therefore, to guarantee the stability of the system before implementing the ILC, a reduced-order mixed-sensitivity $\mathrm{H} \infty$ robust controller was implemented. Due to the use of a small embedded system such as an 8-bit microcontroller, the controller algorithm was designed to be simple enough to run on the system. A traditional synthesis of an $\mathrm{H} \infty$ controller usually generates high order of controller [22]. In this paper, a reduced-order of $\mathrm{H} \infty$ controller combined with iterative learning controller is proposed.

\section{RESEARCH METHOD}

\subsection{Mechanical design}

Mechanical design and the prototype of selective compliance assembly robot arm (SCARA) are shown in Figure 1. The SCARA robot has 4 degrees of freedom with 3 rotational joints and 1 prismatic joint. DC motor on the base or 1st actuator is PG45 dc motor, the 2nd actuator uses PG28 dc motor, the 3rd and the 4th actuator use GA125 dc motor, and lastly, the gripper uses RC servo motor. The Denavit Hartenberg of the SCARA robot is shown in Table 1.

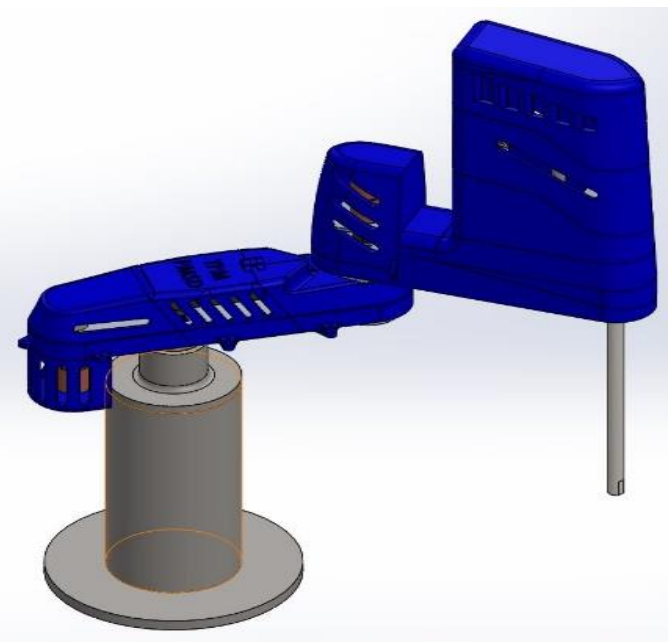

(a)

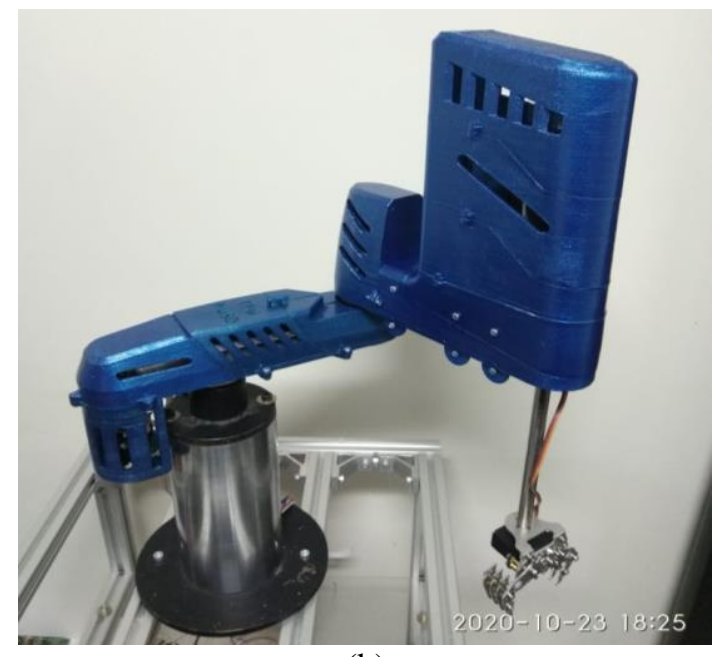

(b)

Figure 1. These figures are, (a) SCARA robot design, (b) SCARA prototype 
Table 1. Denavit Hartenberg table of 4DOF SCARA robot [23]

\begin{tabular}{ccccc}
\hline $\mathrm{i}$ & $\alpha_{i-1}$ & $a_{i-1}$ & $d_{i}$ & $\theta_{i}$ \\
\hline 1 & 0 & 0 & 0 & $\theta_{1}$ \\
2 & 0 & $a_{1}$ & 0 & $\theta_{2}$ \\
3 & 0 & $a_{2}$ & $d_{3}$ & 0 \\
4 & 0 & 0 & 0 & $\theta_{4}$ \\
\hline
\end{tabular}

The forward kinematic (1)-(3) as well as the inverse kinematic of the SCARA robot, is presented as [23]:

$$
\begin{aligned}
& x_{\text {pos }}=a_{1} c_{1}+a_{2} c_{12} \\
& y_{\text {pos }}=a_{1} s_{1}+a_{2} s_{12} \\
& z_{\text {pos }}=d_{3} \\
& \theta_{1}=\tan ^{-1}(y / x)-\tan ^{-1}(k 2 / k 1) \\
& \theta_{2}= \pm \cos ^{-1}\left(\frac{x^{2}+y^{2}-a_{1}^{2}-a_{2}^{2}}{2 a_{1} a_{2}}\right) \\
& d_{3}=z \\
& \theta_{4}=\emptyset-\theta_{1}-\theta_{2}
\end{aligned}
$$

\subsection{Electronic design}

The electronics of the robot consist of microcontrollers using Arduino Uno, dc motor drivers using VNH2SP chip, and power supply $12 \mathrm{~V} 10 \mathrm{~A}$. The block diagram of the electronic is shown in Figure 2 . Each Arduino Uno controls two dc motors. The Unos will receive commands from the Arduino Mega through serial communication. The trajectory command is generated in Arduino Mega. The Arduino Mega also gives commands to the RC servo motor to open or close the gripper.

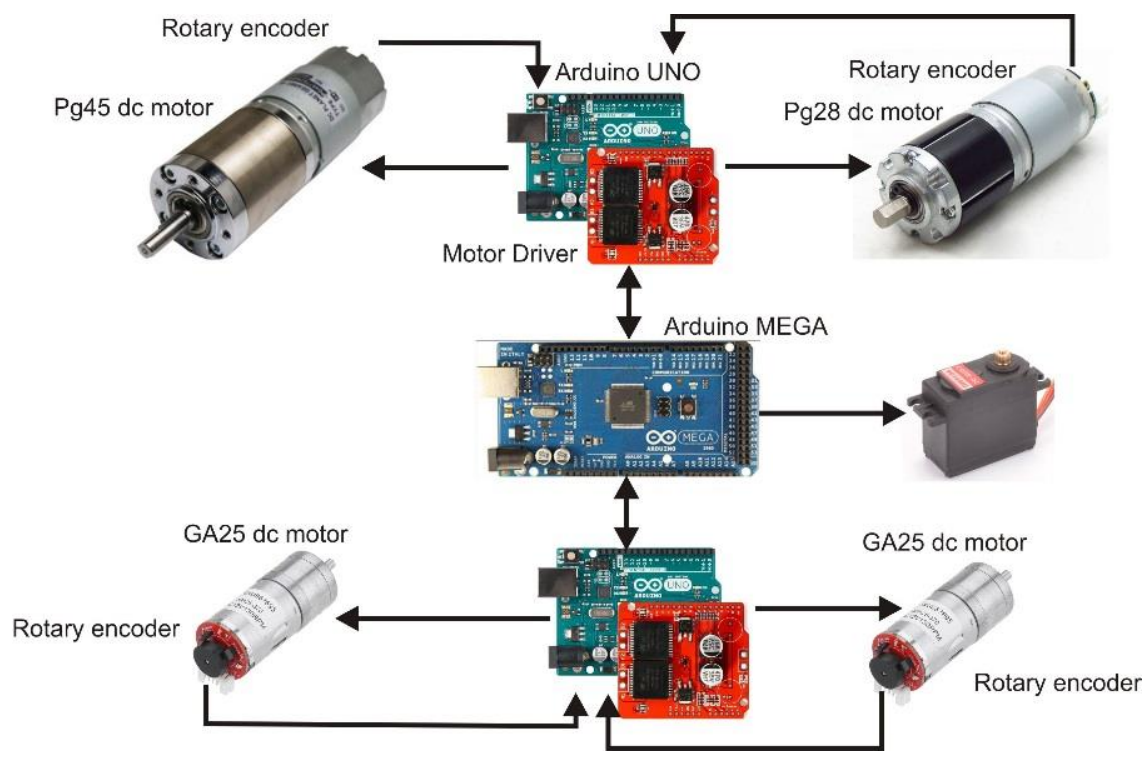

Figure 2. Electronic diagram of the SCARA robot

\subsection{Controller design}

The controller consists of a mixed-sensitivity $\mathrm{H} \infty$ robust controller and iterative learning controller. All the algorithm was written, compiled, and uploaded into an Arduino board. 


\subsubsection{Mixed-sensitivity $H \infty$ robust controller synthesis}

Mixed sensitivity $\mathrm{H} \infty$ shapes the sensitivity function and the complementary sensitivity function of a closed-loop system to get a controller with good performance and robustness. Figure 3 shows a single input single output (SISO) close loop system with multiplicative uncertainty. $G(s)$ is the nominal system, $\Delta(s)$ is the system perturbation, $K(s)$ is the controller, $r(\mathrm{~s})$ is the reference input, $e(\mathrm{~s})$ is the tracking error, $\mathrm{n}(\mathrm{s})$ is the external disturbance, and $y(\mathrm{~s})$ is the output of the system. The controller was designed for $30 \%$ of motor viscous friction and $30 \%$ of load inertia uncertainties.

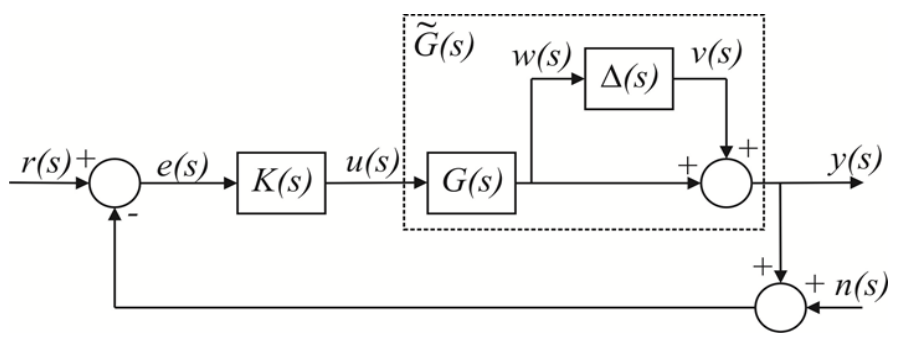

Figure 3. SISO system with multiplicative uncertainties [22]

The perturbed system is expressed by.

$$
\tilde{G}(s)=G_{n}(s)(1+\Delta(s))
$$

The multiplicative system perturbation is:

$$
\Delta(s)=\left(\frac{\tilde{G}(s)}{G_{n}(s)}-1\right)
$$

The mixed-sensitivity $\mathrm{H} \infty$ robust controller was synthesized using the Matlab command "Mixsyn". Mixsyn computes a controller that minimizes the Ho norm of the weighted closed-loop transfer function.

$$
\begin{gathered}
\left\|W_{s}(s) S(s)\right\|_{\infty} \\
\left\|W_{t}(s) T(s)\right\|_{\infty}
\end{gathered}
$$

A known stable function $\mathrm{Ws}(\mathrm{s})$ upper bound the multiplicative perturbation to attenuate external disturbance. Figure 4 shows the function Ws(s) upper-bounds the perturbations.

$$
\Delta(s)_{\infty} \leq W_{t}(s)_{\infty}
$$

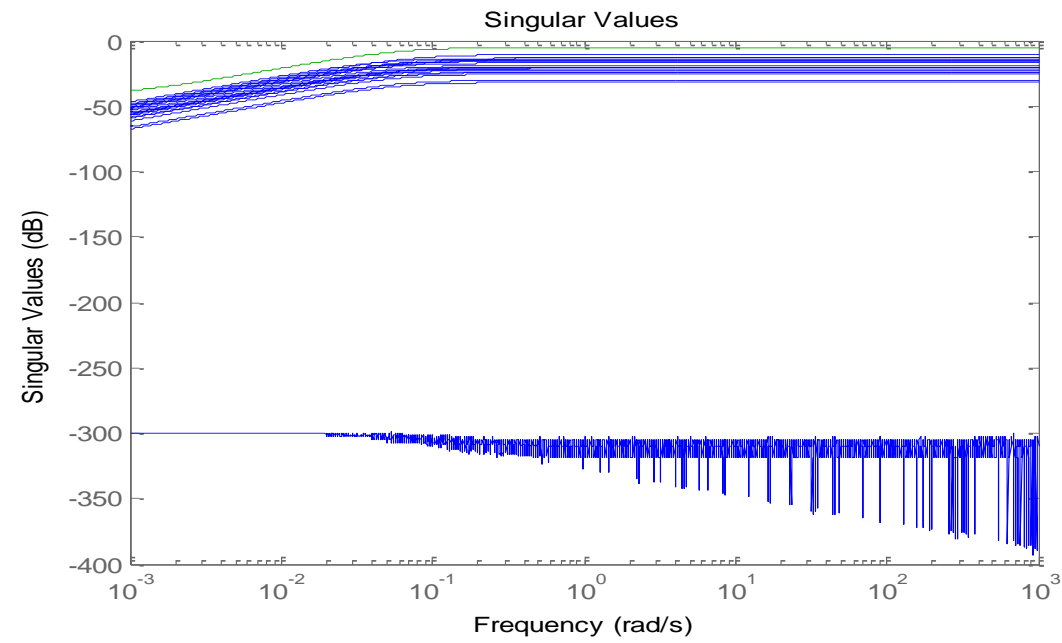

Figure 4. A stable function Ws(s) upper-bounds the perturbations

Robotic arm joint position control using iterative learning and mixed sensitivity $H \bowtie$... (Petrus Sutyasadi) 
Using Skogestad's method [24], the sensitivity weights $\left(W_{s}(s)\right)$ was designed is being as:

$$
W_{s}(s)=\frac{0.5 s+1}{s+0.001}
$$

Figure 5 shows that $|\mathrm{Ws} S|<1$ and $|\mathrm{Wt} \mathrm{T}|<1$ because the singular values of the inverse of the weight functions are larger than the sensitivity and the complementary sensitivity singular values. Matlab control synthesis generate a 3rd order of controller:

$$
K c=\frac{1.132 e 08 s^{2}+1.38 e 07 s+4.202 e 05}{s^{3}+1.181 e 04 s^{2}+8498 s+321}
$$

By using Matlab controller order reducer, a 2nd order of controller was generated from (14):

$$
K c=\frac{1.132 e 08 s+9.499 e 06}{s^{2}+1.181 e 04 s^{2}+8056}
$$

Figure 6 shows by simulation that the proposed controller can control the plant satisfactorily even in the presence of uncertainties from $30 \%$ variation of motor viscous friction and $30 \%$ variation of load inertia.

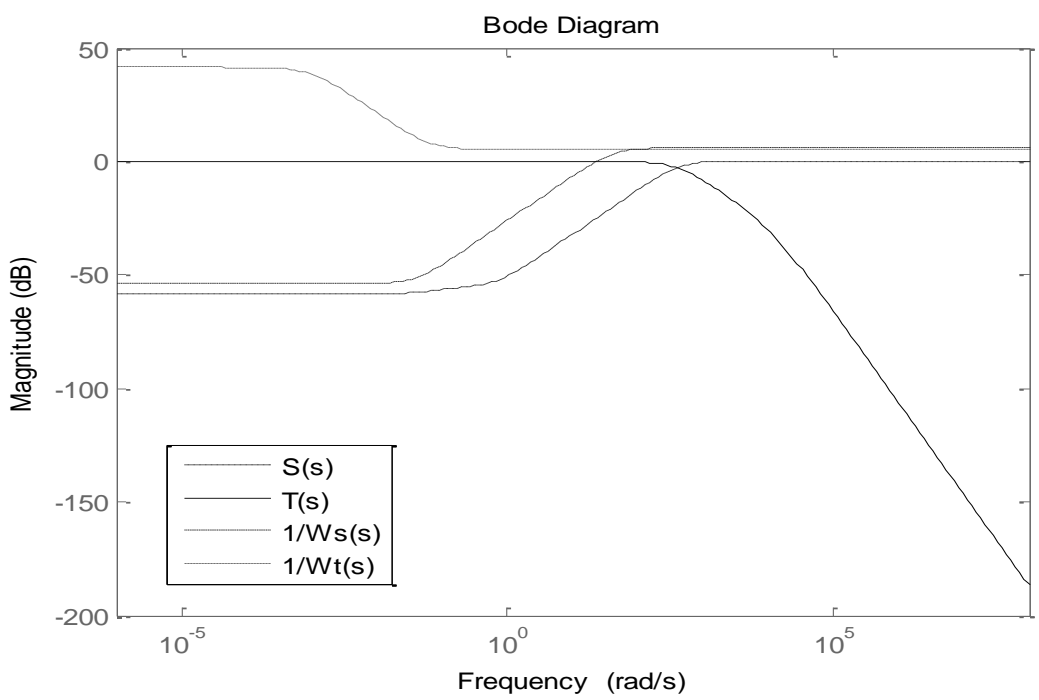

Figure 5. The singular plot of Ws, Wt, S, and T

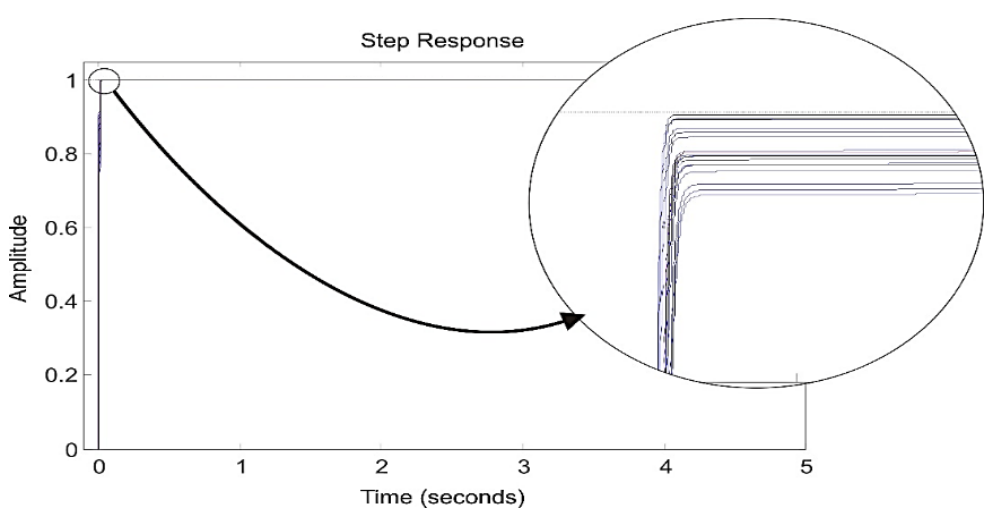

Figure 6. Simulation of mixed sensitivy $H_{\infty}$ robust controller system response 


\subsubsection{Iterative learning control}

By learning the error from the previous trajectory track, iterative learning control (ILC) improves the trajectory tracking of the control system. In using the ILC, several conditions must meet these criteria:

- The trajectory should be a repetitive task.

- The repetitive track should have the same starting and ending position.

- The system should be a stable system before implementing the ILC.

- The tracking performance is improved from one repetition to the next repetition.

- The system should be stable before the ILC is implemented. This is done by the mixed sensitivy $H_{\infty}$ robust controller.

ILC adjusts the manipulated control to follow the trajectory command [25]. The equation of the ILC is determined from.

$$
u_{j}=u_{j-1}+k_{d} \dot{e}_{j-1}(t)+k_{p} e_{j-1}(t)
$$

The variables are:

$u_{j}$ : ILC control signal

$e_{j}$ : error signal

$j$ : iteration number

$k_{p}$ : proportional gain of ILC

$k_{d}$ : derivative gain of ILC

It is a PD type ILC. The structure was chosen because besides simple, according to Xukun [26], this structure is already better than a D type or improved D type ILC. The overall hybrid controller output is the output of mixed sensitivy $H_{\infty}$ robust controller plus the output of ILC. The block diagram of the mixed sensitivy $H_{\infty}$ robust controller-ILC controller is shown in Figure 7. The step-by-step of the overall strategy is shown in Figure 8.

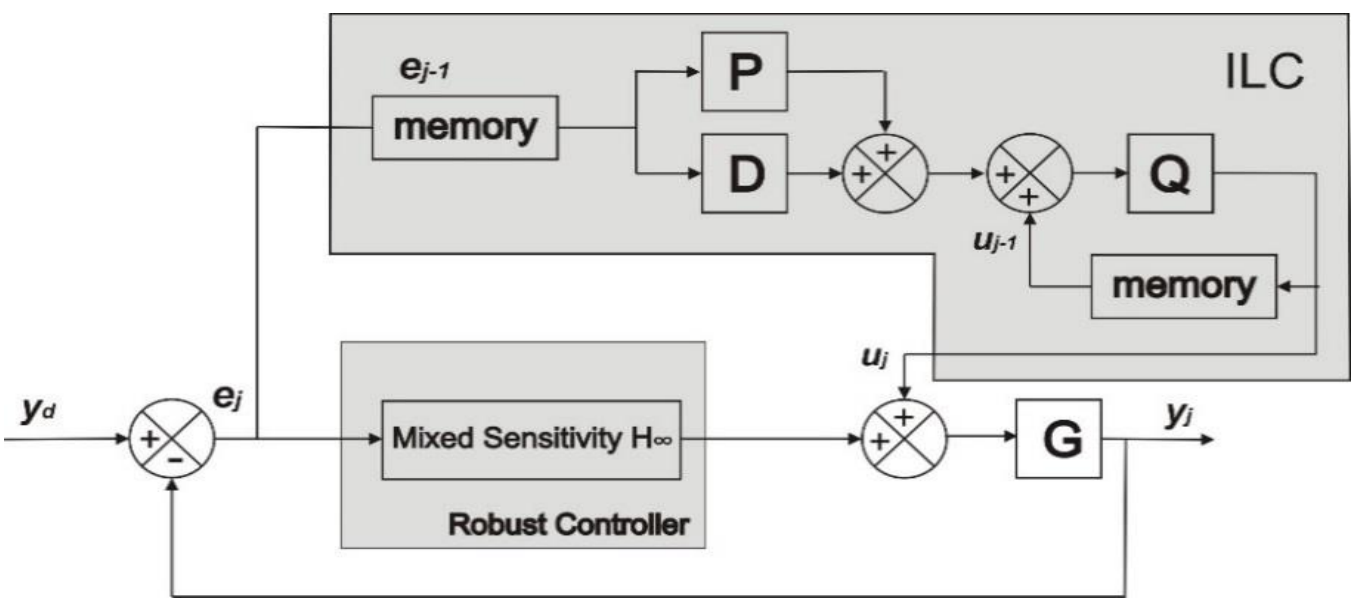

Figure 7. Block diagram of hybrid mixed sensitivy $H_{\infty}$ robust controller-ILC

By manual tuning, the ILC constants were $\mathrm{KP}_{\mathrm{ILC}}=0.015$ and $\mathrm{KD}_{\mathrm{ILC}}=0.01$. Thus, the equation of the ILC becomes.

$$
u_{j}=u_{j-1}+0.01 \dot{e}_{j-1}(t)+0.015 e_{j-1}(t)
$$


Define the nominal model and the uncertainites

Define the Sensitivity and Complementary Sensitivity Weight

Generate the robust controller and reduce the order using Matllab

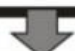

Synthesize an Iterative Learning Controller (ILC)

S

Add Iterative Learning Controller output to the robust controller output

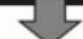

Adjust the ILC gain to get the best response of the proposed hybrid controller

Figure 8. Flowchart of the hybrid controller synthesis steps

\section{RESULTS AND DISCUSSION}

A sinusoidal trajectory was prepared to test the algorithm. The frequency of the signal is $0.5 \mathrm{~Hz}$ and has the span of trajectory command from 0 to 200 degrees. The mixed sensitivy $H_{\infty}$ robust controller response for the sinusoidal input trajectory is shown in Figure 9. The output response was recorded using the attached rotary encoder on each motor. The trajectory was tracked properly. However, it has significant errors along the trajectory. This error along trajectory command is common due to the high inertia of the system. But this can not be accepted for a robotic arm. The error along the slopes should be very small or zero.

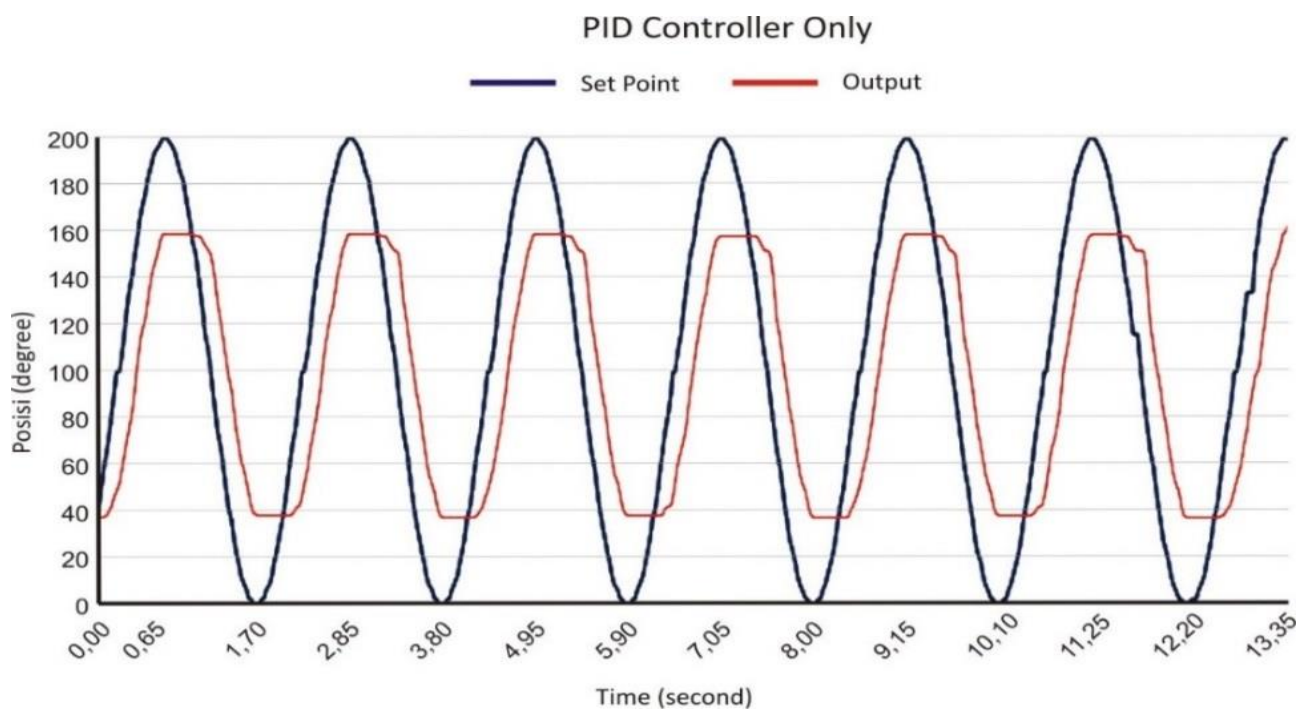

Figure 9. Mixed sensitivity $H_{\infty}$ robust controller response for a sinusoidal input trajectory

The tracking performance was improved by adding iterative learning controller. The hybrid controller performance is shown in Figure 10. After several iterations, the system able to improve its tracking performance. Without ILC, the tracking performance showed in Figure 9 was so poor. In Figure 10, the tracking performance to reach the peak of the setpoint which is positive 200 degrees had around 20 degrees of error. On the second attempt, the output had a better trajectory tracking error around 2 degrees. However, on the second repetition, the system response performance swang back to around 10 degrees. From the third repetition onward, the tracking performance of the system began more stable with the average of error 
tracking around 4 degrees. The system can follow the trajectory command along the slopes of the sinusoidal input. However, there is still around 4 degrees of error on the peak of the trajectory due to the fast change of the direction.

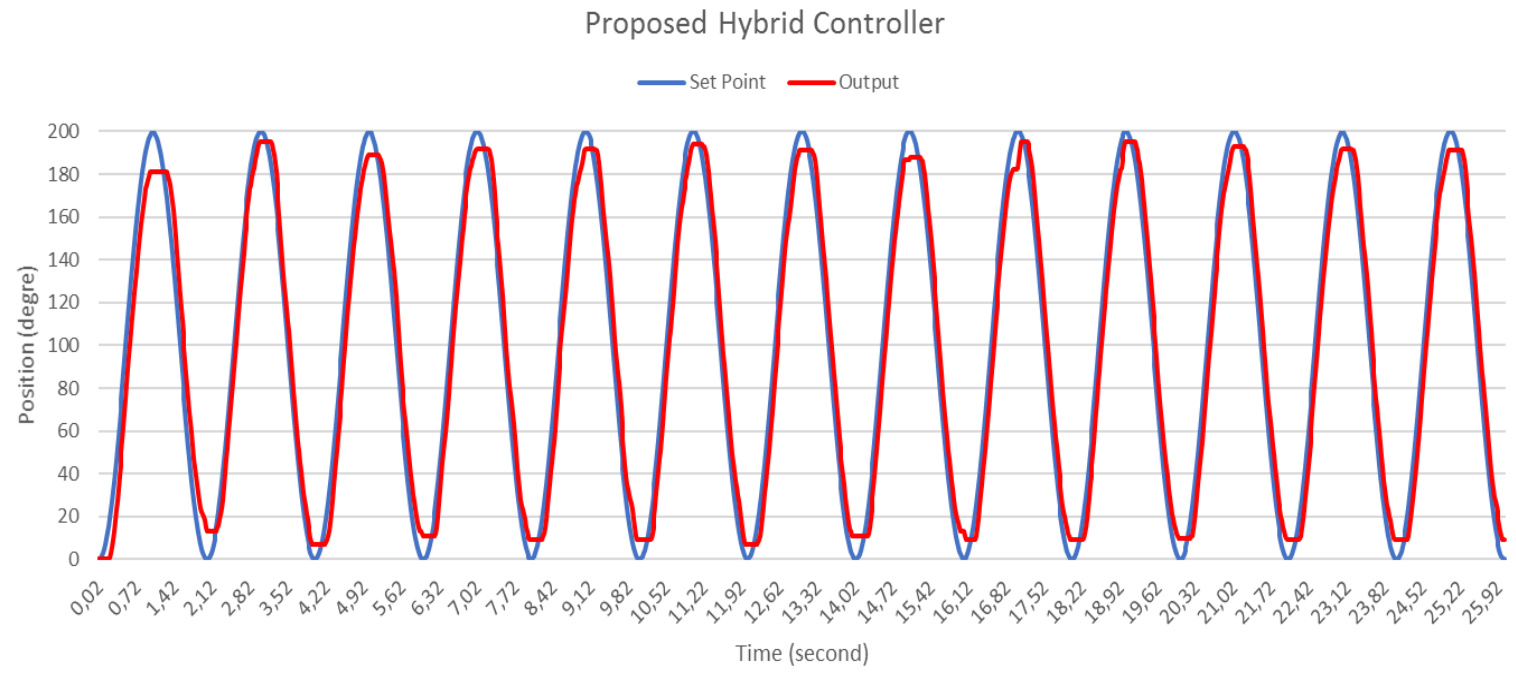

Figure 10. Mixed sensitivity $H_{\infty}$ robust controller combined with iterative leaning controller response for a sinusoidal input trajectory

The proposed controller performance was compared to the previous robot joint controller which was a PID cascade controller. The PID cascade controller was designed to have a position control loop and speed control loop. Figure 11 shows the performance of the PID cascade loop. The PID cascade loop can adjust its speed besides its position control. Therefore, for a high-frequency sinusoidal input, it can reduce the response lag time and improve the error along the slopes of the trajectory. At the peak of the trajectory command, the PID cascade has a big error around 15 degrees. The cascade controller could be adjusted for higher gain to reduce the error, but consequently, the response became very sensitive to input disturbance. The PID cascade output response is not smooth in higher gain.

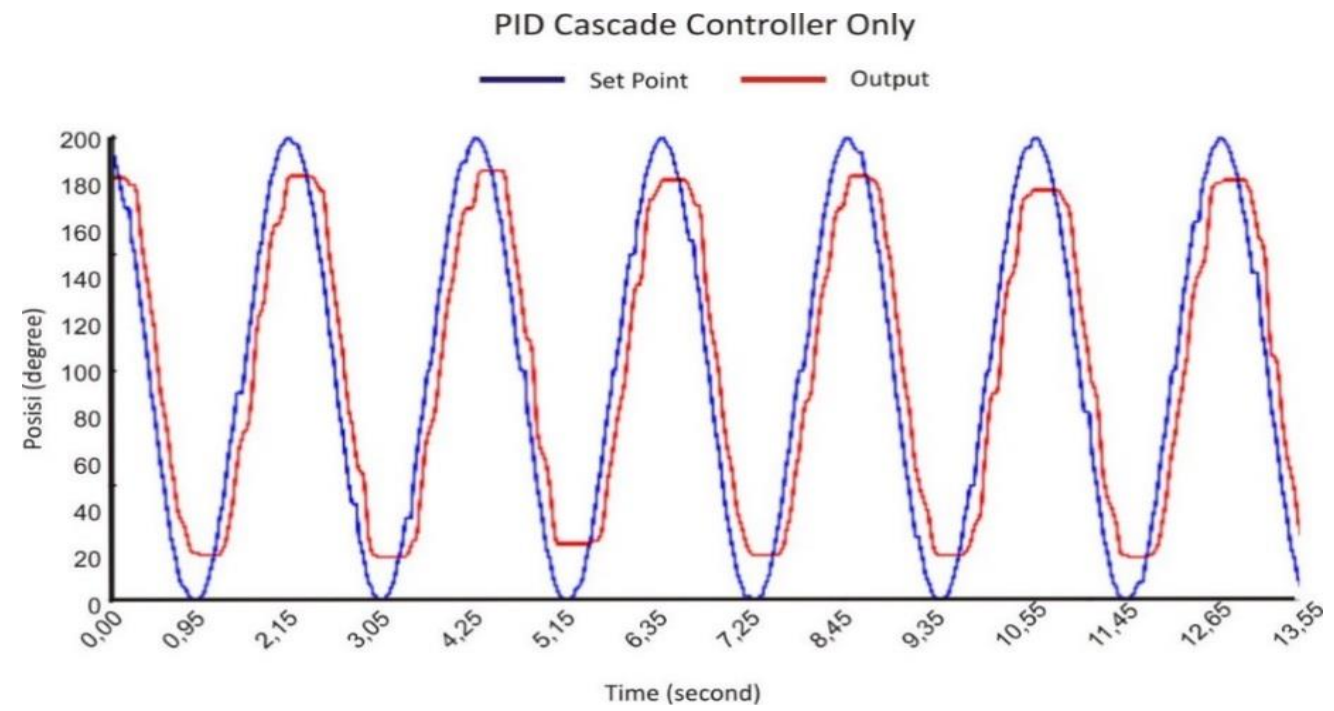

Figure 11. PID cascade controller or previous robot joint control algorithm response for a sinusoidal input trajectory 


\section{CONCLUSION}

This study aimed was to design and investigate a hybrid controller of mixed sensitivy $H_{\infty}$ robust controller with iterative learning controller for controlling a joint position of a low-cost robotic arm. The Investigation concluded that the proposed controller was able to track the trajectory better than the previous robot control algorithm which was a PID cascade controller. The relevance of the work is supported by the current findings that the proposed controller has no errors in tracking the sinusoidal trajectory command, except on the fast-changing of the direction, it has around 4 degrees of lagging response. This was due to the fast-changing of the direction of the trajectory command. However, this is still better than the PID cascade controller that has around 15 degrees of similar lagging response and always has error along with the trajectory command. The advancement reaches around $73,33 \%$ from 15 to 4 degrees in reaching the positive or negative peak of the setpoint. These findings enhance our understanding that the mixed sensitivy $H_{\infty}$ Robust controller can guarantee stability over the uncertainties for example the load variation and the iterative learning controller will fix the limitation of the tracking performance of the robust controller over time along with the repetition. The tracking performance of the mixed sensitivy $H_{\infty}$ robust controller will not be the best tracking because the controller works as an optimal controller that provides the optimal performance in the range of uncertainties [27]. Therefore, its tracking performance was designed to be improved by the ILC.

\section{ACKNOWLEDGEMENTS}

The authors would like to acknowledge the research funding support from the Ministry of Research, Technology, and Higher Education of the Republic of Indonesia (Kemen-RISTEKDIKTI).

\section{REFERENCES}

[1] A. Weber, "Robotics in the Age of Industry 4.0," Assembly, vol 63, no. 5, pp. 64-70, May 15. 2020.

[2] WEF, "The Future of Jobs. Employment, Skills and Workforce Strategy for the Fourth Industrial Revolution," Global Challenge Insight Report, Davos, Switzerland, 2016.

[3] IFR. World Robotics. Industrial Robots 2014; Frankfurt, M., Ed.; International Federation for Robotics: New York, NY, USA, 2015

[4] M. Ceccarelli, "Low-Cost Robots for Research and Teaching Activities," IEEE Robotics \& Automation Magazine, 2001, doi: 10.1109/mra.2003.1233556.

[5] P. Sutyasadi and M. Parnichkun, "Gait Tracking Control of Quadruped Robot Using Differential Evolution Based Structure Specified Mixed Sensitivity Robust Control," Journal of Control Science and Engineering, vol. 2016, doi: 10.1155/2016/8760215.

[6] Y. V. Hote, "PI controller design for one joint robotic arm," 2016 14th International Conference on Control, Automation, Robotics and Vision (ICARCV), 2016, pp. 1-6, doi: 10.1109/ICARCV.2016.7838714.

[7] S. C. Suhaimin, N. L. Azmi, M. M. Rahman, and H. M. Yusof, "Analysis of Point-to-Point Robotic Arm Control using PID controller," 2019 7th International Conference on Mechatronics Engineering (ICOM), 2019, pp. 1-6, doi: 10.1109/ICOM47790.2019.8952036.

[8] M. U. Almerich, 'PID Control for Robotic Arm,'” Final degree project, Universitat Politècnica de València, 2020.

[9] D. Zhang and B. Wei, "Design of a joint control system for serial mechanical arms based on PID and MRAC control," 2016 Asia-Pacific Conference on Intelligent Robot Systems (ACIRS), 2016, pp. 91-96, doi: 10.1109/ACIRS.2016.7556194.

[10] R. N. Lea, J. Hoblit, and Y. Jani, "Fuzzy logic based robotic arm control," [Proceedings 1993] Second IEEE International Conference on Fuzzy Systems, 1993, pp. 128-133 vol.1, doi: 10.1109/FUZZY.1993.327451.

[11] S. Tomas, K. Michal, and K. Alena, "Fuzzy control of robotic arm implemented in PLC," 2013 IEEE 9th International Conference on Computational Cybernetics (ICCC), 2013, pp. 45-49, doi: 10.1109/ICCCyb.2013.6617628.

[12] G. M. Khoury, M. A. Saad, H. Y. Kanaan, and C. Asmar, "Fuzzy PID Control of a Five DOF Robot Arm," Journal of Intelligent and Robotic Systems, vol. 40, no. 3, pp. 299-320, 2004, doi: 10.1023/B:JINT.0000038947.97195.22.

[13] H. A. Malki, D. Misir, D. Feigenspan, and G. Chen, "Fuzzy PID control of a flexible-joint robot arm with uncertainties from time-varying loads," in IEEE Transactions on Control Systems Technology, vol. 5, no. 3, pp. 371-378, May 1997, doi: 10.1109/87.572133.

[14] D. P. Nam, N. H. Quang, T. P. Nam, and T. T. H. Yen, "Adaptive dynamic programming based optimal control for a robot manipulator," International Journal of Power Electronics and Drive System (IJPEDS), vol. 11, no. 3, pp. 1123-1131, September 2020, doi: 10.11591/ijpeds.v11.i3.

[15] W. S. Pambudi, E. Alfianto, A. Rachman, and D. P. Hapsari, "Simulation design of trajectory planning robot manipulator," Bulletin of Electrical Engineering and Informatics (BEEI), vol. 8, no. 1, pp. 196-205, March 2019, doi: 10.11591/eei.v8i1.1179.

[16] K. S. Gaeid, A. F. Nashee, I. A. Ahmed, and M. H. Dekheel, "Robot control and kinematic analysis with 6DoF manipulator using direct kinematic method,' Bulletin of Electrical Engineering and Informatics (BEEI), vol. 10, no. 1, pp. 70-78, February 2021, doi: 10.11591/eei.v10i1.2482. 
[17] T. Soehartanto, I. F. Imran, and L. A. Purwitosari, "Control design for direct-drive robotic ARM using sliding mode control," 2017 International Conference on Advanced Mechatronics, Intelligent Manufacture, and Industrial Automation (ICAMIMIA), 2017, pp. 277-282, doi: 10.1109/ICAMIMIA.2017.8387601.

[18] Trong-Thang Nguyen, "Sliding mode control-based system for the two-link robot arm," International Journal of Electrical and Computer Engineering (IJECE), vol. 9, no. 4, pp. 2771-2778, August 2019, doi: 10.11591/ijece.v9i4.pp2771-2778.

[19] S. A. Ajwad, R. U. Islam, M. R. Azam, M. I. Ullah, and J. Iqbal, "Sliding mode control of rigid-link anthropomorphic robotic arm," 2016 2nd International Conference on Robotics and Artificial Intelligence (ICRAI), 2016, pp. 75-80, doi: 10.1109/ICRAI.2016.7791232.

[20] T. L. Nguyen and H. T. Vu, "Super-twisting sliding mode based nonlinear control for planar dual arm robots," Bulletin of Electrical Engineering and Informatics, vol. 9, no. 5, pp. 1844-1853, October 2020, doi: 10.11591/eei.v9i5.2143.

[21] A. A. Mohammed and A. Eltayeb, "Dynamics and Control of a Two-link Manipulator using PID and Sliding Mode Control," 2018 International Conference on Computer, Control, Electrical, and Electronics Engineering (ICCCEEE), 2018, pp. 1-5, doi: 10.1109/ICCCEEE.2018.8515795.

[22] P. Sutyasadi and M. Parnichkun, "Push recovery control of quadruped robot using particle swarm optimization based structure specified mixed sensitivity $\mathrm{H} 2 / \mathrm{H} \infty$ control," Industrial Robot: the international journal of robotics research and application, vol. 47, no. 3, pp. 423-434, 2020, doi: 10.1108/IR-06-2019-0135.

[23] Adapala Academy \& IES GS for Exams, 3.7 Robotics || ESE Mains \| Inverse kinematics of SCARA robot, Jan. 31, 2020. Accessed on Apr. 11, 2021. [Video file]. Available: https://www.youtube.com/watch?v=yfhYGrGbDBw.

[24] S. Skogestad and I. Postlethwaite, Multivariable Feedback Control Analysis and Design, New York, NY, USA: Wiley, 2001, pp. 789-794.

[25] P. Sutyasadi and M. Parnichun, "Trotting control of a quadruped robot using PID-ILC," IECON 2015 - 41st Annual Conference of the IEEE Industrial Electronics Society, 2015, pp. 004400-004405, doi: 10.1109/IECON.2015.7392784.

[26] G. Xukun, W. Lin, and Z. Zidong, "Trajectory Tracking of Manipulator Based on Iterative Learning Control," 2020 IEEE International Conference on Advances in Electrical Engineering and Computer Applications (AEECA), 2020, pp. 1009-1015, doi: 10.1109/AEECA49918.2020.9213540.

[27] P. Sutyasadi and M. B. Wicaksono, "Joint Control of a Robotic Arm Using Particle Swarm Optimization Based $\mathrm{H} 2 / \mathrm{H} \infty$ Robust Control on Arduino," TELKOMNIKA Telecommunication, Computing, Electronics and Control, vol. 18, no. 2, pp. 1021-1029, 2020, doi: 10.12928/TELKOMNIKA.v18i2.14749

\section{BIOGRAPHIES OF AUTHORS}

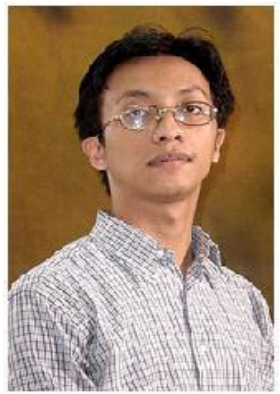

Petrus Sutyasadi earned his master degree in mechatronics in 2008 and doctoral degree in mechatronics in 2016 from the Asian Institute of Technology, Thailand. Currently he is a lecturer at Mechatronics Department of Politeknik Mekatronika Sanata Dharma, Yogyakarta, Indonesia. His research interest includes robust control, robotic, and frugal innovation in mechatronic engineering.

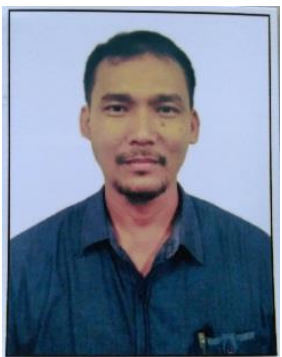

Martinus Bagus Wicaksono earned his master degree in Design and Manufacturing Engineering in 2011 from the Asian Institute of Technology, Thailand. Currently, he is a lecturer at Mechanical Design Technology of PMSD, Yogyakarta, Indonesia. His research interest includes rapid prototyping and advanced Manufacturing. 